\title{
Questes
}

Revue pluridisciplinaire d'études médiévales

\section{La scatologie dans Audigier : de la chanson de geste au fabliau}

\section{Chloé Chalumeau}

\section{(2) OpenEdition}

Journals

\section{Édition électronique}

URL : http://journals.openedition.org/questes/2654

DOI : 10.4000/questes.2654

ISSN : 2109-9472

\section{Éditeur}

Les Amis de Questes

\section{Édition imprimée}

Date de publication : 15 juin 2011

Pagination : 55-71

ISSN : 2102-7188

\section{Référence électronique}

Chloé Chalumeau, «La scatologie dans Audigier: de la chanson de geste au fabliau », Questes [En ligne], 21 | 2011, mis en ligne le 15 janvier 2014, consulté le 01 mai 2019. URL : http:// journals.openedition.org/questes/2654; DOI : 10.4000/questes.2654 


\section{La scatologie dans Audigier : de la chanson de geste au fabliau}

\section{Chloé CHALUMEAU}

Vraisemblablement composé à la fin du XII ${ }^{\mathrm{e}}$ siècle ou au tout début du XIII ${ }^{\mathrm{e}}$, Audigier a longtemps dérouté une critique prompte à s'effaroucher de son débordement ordurier. Il est vrai que si la chanson, qui narre les aventures du comte Turgibus et de son fils, emprunte une forme et des thématiques épiques, c'est pour les situer dans un véritable cloaque : tout y est prétexte à évoquer les excréments, de la conception du héros à son mariage final en passant par ses exploits guerriers. La disparité entre la forme du poème, qui rappelle indubitablement la chanson de geste, et sa matière, qui l'oriente nettement vers le fabliau, invite à s'interroger sur la spécificité de la scatologie dans ce texte. Qu'a-t-elle à voir avec les poèmes épiques? Est-elle semblable à celle des fabliaux ? L'indécision générique d'Audigier peut-elle être révélatrice d'une évolution littéraire? Après avoir montré qu'Audigier se situe au confluent des deux genres, nous essaierons de comprendre quelle vision du monde véhicule ici la scatologie. En effet, la coexistence de motifs nobles et d'un registre bas conduit à voir dans le texte une dégradation du genre épique, propice à l'expression d'une idéologie carnavalesque qui est aussi à l'œuvre dans les fabliaux. La discordance comique entre forme et matière semble également introduire une réflexion sur la littérature et le langage qui n'est pas non plus étrangère à ces textes. Cependant, la poétique du poème ne peut se confondre avec celle du fabliau. Situé dans une zone intermédiaire inédite entre deux genres, sorte d'hapax dans la littérature médiévale, que peut nous apprendre Audigier? 


\section{De la forme épique à la matière du fabliau}

Ressort nettement, à la lecture d'Audigier, la discordance entre la forme et le sujet du poème, à l'évidence inspirés de la chanson de geste, et son contenu, qui appartient entièrement au registre bas, presque exclusivement scatologique: si Audigier chante en décasyllabes les exploits guerriers et amoureux de Turgibus et de son fils, ces deux derniers passent la majeure partie du texte à produire et recevoir des excréments, quand ils ne pataugent pas tout simplement dedans. Cette discordance entre une forme noble et un contenu manifestement dégradé a conduit la tradition critique, que commente Omer Jodogne dans son édition du poème ${ }^{1}$, à définir Audigier comme une parodie de chanson de geste et donc à essayer d'en trouver le modèle ${ }^{2}$. Cependant, la diversité des sources possibles et le caractère vague des rapprochements qui servent à les identifier incitent à prendre avec précaution non seulement les résultats de ces recherches mais leur présupposé même : rien n'indique en effet que l'auteur d'Audigier ait voulu parodier un texte en particulier et l'on serait en droit de supposer que, si c'était le cas, il aurait fait en sorte de le rendre immédiatement identifiable à son public. Plutôt que la parodie d'un texte, Audigier serait une réécriture des modèles épiques en général dont il reprendrait le squelette formel et thématique ${ }^{3}$, ce qui remettrait en cause l'idée même de parodie. En effet, d'après les catégories établies par Gérard Genette, la

\footnotetext{
${ }^{1}$ Omer Jodogne, «Audigier et la chanson de geste avec une édition nouvelle du poème », Moyen Âge, 66 (1960), p. 495-526. Les références à Audigier renvoient à cette édition.

2 Des ressemblances ont ainsi été vues avec le Girart de Roussillon d'Oxford, la seconde partie de la Chanson de Guillaume, Aspremont, Floovant, ou encore Aiol.

${ }^{3}$ La structure strophique s'inspire de la chanson de geste romancée de la seconde moitié $\mathrm{du} \mathrm{XII}^{\mathrm{e}}$ siècle et les motifs épiques sont ceux que répertorie Jean Rychner (adoubement, description de l'armement, combats...) auxquels on peut ajouter des motifs qui relèvent de la chanson de geste romancée (généalogie du héros, enfances, noces...). Cf. Jean RYCHNER, La Chanson de geste. Essai sur l'art épique des jongleurs, Genève-Lille, Droz, 1955, p. 128-130.
} 
parodie est la reprise d'un hypotexte précis ${ }^{4}$ et non pas le simple décalage entre la forme noble et le contenu dégradé que la critique a depuis longtemps relevé. Omer Jodogne conclut d'ailleurs : «je ne définirais pas Audigier une parodie de chanson de geste, mais [...] un poème héroïcomique ayant la forme d'une chanson de geste $»^{5}$. N'ayant alors de chanson de geste que la forme, Audigier se rapprocherait du fabliau par la matière, ce que tendrait à confirmer son insertion dans un recueil de fabliaux ${ }^{6}$. De même que notre texte, les fabliaux présentent en effet de nombreuses occurrences du bas corporel excrémentiel, comme en témoignent, entre autres, Jouglet, La Crote, Les Trois Meschines ou le Pez. au vilain ${ }^{7}$. Cependant, la simple parenté de registre et de thèmes scatologiques suffit-elle à rapprocher de manière pertinente Audigier des fabliaux ?

Tout d'abord, écarter Audigier de la chanson de geste en raison de l'omniprésence de thèmes bas paraît aujourd'hui relever d'une vision bien épurée des poèmes épiques, qui versent souvent dans un registre moins soutenu que celui qu'on veut bien leur prêter ; il suffit pour s'en convaincre de se souvenir des exploits de Rainouart à la cuisine dans La Chanson de Guillaume et dans Aliscans ${ }^{8}$, de la mésaventure de Tiébaut au début de $L a$

\footnotetext{
${ }^{4}$ Cf. Gérard GenetTe, Figures I, Paris, Seuil, 1966. Établies à partir des textes de la période classique, les catégories de Genette ne recouvrent pas les spécificités littéraires du Moyen Âge, mais permettent cependant d'éviter la confusion entre différents termes souvent utilisés sans grande précision.

${ }^{5}$ Omer JodoGnE, «Audigier et la chanson de geste... », art. cit., p. 511.

${ }^{6}$ Au sein du manuscrit Paris, BnF, f. fr. 19152.

7 Pour tous les fabliaux, voir le Nouveau Recueil Complet des Fabliaux, Willem NOOMEN et Nico VAN DEN BoOgAaRd (éd.), Assen, Van Gorcum, 1983-1998, 10 tomes.

${ }^{8}$ Pour sa première apparition dans les deux œuvres, Rainouart surgit en effet des cuisines comme un diable de sa boîte, couvert de haillons, maculé de fumée, et combattant les garçons de cuisine dans une scène ouvertement comique. Cf. La Chanson de Guillaume, Philip E. BENNETT (éd. et trad.), Londres, Grant and Cutler Ldt, 2000, v. 2648-2717 et Aliscans. Chanson de geste du XII e siècle, Claude REGNIER (éd.) et Andrée et Jean Subrenat (trad.), Paris, Champion, «Classiques Français du Moyen Âge », 110-111, 1990, v. 3565-3608.
} 
Chanson de Guillaume ${ }^{9}$ ou bien d'Aiol, dans lequel le malheureux héros se fait jeter des ordures au visage avant d'être aux prises avec une bouchère à « le panche grose et le cul grant » qui ressemble fortement à Grimberge, l'ennemie d'Audigier ${ }^{10}$. Dans tous ces textes, les excréments ou leur forme atténuée servent à manifester l'humiliation de chevaliers dont on veut dénoncer la lâcheté. Bien sûr, à la différence de ces textes, Audigier semble ne se complaire que dans le thème scatologique et l'applique à tous ses personnages, mais cette remarque invite à penser que notre poème pourrait pousser dans l'excès et la caricature une tendance déjà présente dans la chanson de geste: la scatologie ne serait ainsi que la représentation concrète de la couardise, amplifiée et exemplifiée jusqu'à l'écœurement dans un texte qui se présente justement comme l'épopée d'un anti-héros «de for lignaige li plus hardiz / et de toz les coarz li plus failliz ${ }^{11}$.

Ensuite, si la scatologie semble prisée aussi des fabliaux, un examen de sa fonction narrative montre qu'elle n'y tient pas du tout le même rôle que dans Audigier. Les fabliaux qui se construisent autour d'un motif scatologique en font un élément majeur de leur schéma narratif : ressort de l'action, l'excrément joue le rôle de l'élément perturbateur dans une situation initiale ordonnée ou du rebondissement inattendu à la fin du texte. Dans Le Pez au vilain par exemple, le pet qui prend la place de l'âme est l'accident autour duquel le fabliau s'organise, tandis que dans La Crote, l'excrément sert le retournement de situation qui permet de clore l'intrigue ${ }^{12}$. Dans Audigier, saturé de flatulences et de défécations en tous

\footnotetext{
${ }^{9}$ «Li uns des penduz li hurte lunc a boche; / vit le Tebald sin out doel et vergoigne, / de la pour en ordead sa hulce. », La Chanson de Guillaume, éd. cit., v. 344-346.

${ }^{10}$ Aiol, Jacques NORMAND et Gaston RAYNAUd (éd.), Paris, SATF, 1877, v. 2689. Aiol répond à Hersent qui lui offre une andouille : «Molt vous aiment ches mousques par Dé le grant, / Car vos estes lor mere, mien ensiant : / Entor vos trevent merde, j'en sai itant, / Que a molt grans tropiaus vos vont sivant » (v. 2710-2713).

${ }^{11}$ Audigier, éd. cit., v. 105-106.

${ }^{12}$ Voir dans ce bulletin la contribution de Francesco MONTORSI, «Quelques pistes de réflexions pour une étude scatologique », p. 35-54.
} 
genres, on ne peut guère donner de fonction dans le schéma narratif qu'à l'affront de Grimberge qui déclenche les hostilités lors de l'adoubement d'Audigier :

Et, por lui faire honte corroucier

Se descouvri la dame sanz atargier,

Tres enmi les quaroles ala chier.

Molt en pesa forment a Audigier $;{ }^{13}$

Peut-être les humiliations excrémentielles infligées par la vieille femme, parce qu'elles consacrent la défaite du héros, sont-elles aussi à interpréter comme ayant une fonction narrative, mais force est de remarquer qu'elles n'introduisent aucun retournement de situation. Dans tous les autres cas, les excréments semblent moins jouer un rôle précis dans le récit qu'en remplacer tous les autres éléments : s'ils ont une fonction à remplir, il s'agit proprement d'une fonction de substitution en vertu de laquelle ils peuvent faire aussi bien office d'adversaires ( «Uns estrons l'abati par meschëance ») que de sièges («Les napes estendirent sor estronz sés »), servir de nourriture (« ses doiz boute en la merde, puis si les suce») ou d'argent pour payer les jongleurs (« et Audigier lor donne molt lieement : / trente crotes de chievre a chascun tent. ») ${ }^{14}$. Les excréments ne sont donc pas liés à la ruse comme dans les fabliaux et ne servent ni les coups de théâtre ni les rebondissements narratifs. Dessinant une toile de fond malodorante dans la contrée de «Cocuce, un païs mou / ou les genz sont en merde jusques au cou ${ }^{15}$, ils semblent plus envahir les motifs que les constituer réellement.

S'il reprend un registre et une thématique typiques des fabliaux, Audigier n'en fait donc pas le même usage, et la tonalité épique du texte l'éloigne aussi des fabliaux à thématique ordurière auxquels elle est totalement étrangère. C'est cette tonalité épique, en complet décalage avec

\footnotetext{
${ }^{13}$ Audigier, éd. cit., v. 216-219.

${ }^{14}$ Ibid., v. 31 ; v. 85 ; v. 13 ; v. 515-516.

${ }^{15}$ Ibid., v. 3-4.
} 
le contenu de la chanson, qui permet à Omer Jodogne de définir Audigier comme un «poème héroï-comique ${ }^{16}$. Cependant, l'héroïcomique a pour principe d'élever un sujet vulgaire. Audigier repose-t-il sur l'épicisation d'une matière basse, ou au contraire sur la dévalorisation d'une matière noble ? Il semble justement que si les excréments remplacent les données traditionnelles des récits héroïques et se substituent aux motifs classiques, c'est que ces données et ces motifs sont premiers : les remplacer, qui plus est par de la fange, c'est procéder à un évidement, à un systématique rabaissement de la matière. Audigier semble ainsi se moquer, en les subvertissant, des motifs épiques classiques, et donc se rapprocher du burlesque des fabliaux et de l'idéologie qu'il met en œuvre.

\section{Une idéologie carnavalesque}

Ce rabaissement systématique de ce qui est élevé rattache Audigier au carnavalesque, le fameux « triomphe de l'affranchissement provisoire de la vérité dominante et du régime existant, d'abolition provisoire de tous les rapports hiérarchiques, privilèges, règles et tabous ${ }^{17}$ de Bakhtine. Audigier, en pervertissant la matière épique traditionnelle, manifesterait ainsi la transgression des valeurs dont cette littérature est le reflet et se tournerait ostensiblement vers l'instauration du désordre. En effet, la chanson construit au pays de Cocuce un véritable mundus inversus, dans lequel la perversion des valeurs chevaleresques semble condensée dans la reprise parodique du motif de l'osculum, véritable fil conducteur du poème : introduit par la défaite de Turgibus contre un étron auquel il doit rendre hommage ${ }^{18}$ ce motif subit ensuite une inversion flagrante lorsque,

\footnotetext{
${ }^{16}$ Omer JODOGNE, « Audigier et la chanson de geste... », art. cit., p. 495.

${ }^{17}$ Mikhaïl BAKHTINE, L'CEuvre de François Rabelais et la culture populaire au Moyen Âge et sous la Renaissance, Paris, Gallimard, 1970 ( $1^{\text {ère }}$ éd. russe 1965), p. 18-19.

${ }^{18}$ « homaige fist l'estront par sa fiance, / puis le baisa trois foiz par racordance : / issi fu la pais faite et l'alïance. », Audigier, éd. cit., v. 34-36.
} 
semblable au chevalier de Bérangier au long cul, Audigier se voit à plusieurs reprises contraint d'embrasser le derrière de Grimberge, ce qui donne lieu à un échange de la face et du derrière typiquement carnavalesque :

deus foiz li fist baisier son cul ainz qu'il fust ters et Audigier i ert par ses lievres aers. « Audigier, dist Grinberge, mes cus est ters. » Grainberge est descouverte jusqu'au nombriz, sor Audigier s'asiet non pas enviz, sor sa face li a son cul assis. ${ }^{19}$

L'inversion des rapports hiérarchiques entre homme et femme, que la victoire de Grimberge sur Audigier rend d'autant plus patente qu'elle est spatialement signifiée, est le signe d'un désordre qui ne se borne pas aux motifs épiques mais s'étend plus largement aux valeurs du monde médiéval. Les rituels amoureux font ainsi les frais de la dérision scatologique, comme le montrent les fiançailles d'Audigier et de Troncecrevasse $^{20}$, et la religion elle-même devient l'objet d'un traitement irrévérencieux dont témoignent le baptême du héros ${ }^{21}$ et l'inversion des motifs hagiographiques que sont les effets miraculeux de la dépouille de Turgibus $^{22}$. En créant un monde renversé qui, significativement, échappe au regard divin ( «ce est une contree ou Dieus n'est mie ${ }^{23}$ ), la chanson d'Audigier se rapproche de l'univers temporairement désordonné du carnaval.

Cependant, elle instaure paradoxalement au sein de sa narration un certain ordre qui, s'il n'est pas narratif, est au moins actanciel, puisqu'il

\footnotetext{
${ }^{19}$ Ibid., v. 415-420.

${ }^{20}$ «si l'en a enmenee en un prael, / puis la fist acroupir enz el plus bel; / en chiant li a mis el doi l'ennel. », ibid., v. 487-489.

${ }^{21}$ Le baptême a lieu «soz un seillier, / la ou dame Raimberge soloit pissier », ibid., v. 155-156.

22 «toutes choses i vont por enpoirier, / nes li chien du païs i vont pissier / qu'il i cuident guarir de l'enragier », ibid., v. 116-118.

${ }^{23}$ Ibid., v. 26.
} 
sert à distinguer adjuvants et opposants. Cet ordre interne au texte se fonde sur des valeurs qui lui sont externes, et qui viennent ainsi nier en partie le caractère subversif d'Audigier. Kathryn Gravdal montre avec justesse qu'à l'intérieur même du désordre scatologique apparait une opposition entre «vilain» et «courtois », symbolisée par la création dans le texte d'un espace bipartite, tendu entre le monde civilisé du comte Audigier et celui presque sauvage de la paysanne Grimberge ${ }^{24}$. Suivant cette dichotomie sociale, les excréments «négatifs » de Grimberge et de ses filles viennent bouleverser les excréments «positifs» du monde courtois, avant que la scatologie ne retrouve son statut positif initial et que le mariage du héros ne marque le retour à l'ordre final.

Paradoxalement, Audigier s'appuie donc sur les valeurs traditionnelles qui associent la figure méprisée du vilain aux excréments, thème récurrent de la littérature médiévale, largement exploité par les fabliaux ${ }^{25}$. L'originalité du texte, cependant, consiste à faire patauger dans la même substance nauséabonde toutes les catégories sociales, frappant ainsi l'ordre actanciel d'artificialité : comment voir un ordre quelconque dans l'histoire d'un chevalier couard, né dans les excréments, défait par des femmes fangeuses et qui se marie au milieu des étrons? Alors que le fabliau se construit très nettement sur un schéma narratif qui conduit de l'instauration d'un désordre à un retour à l'ordre (au moins apparent), Audigier paraît reposer sur un désordre qui n'est jamais résolu puisque Audigier s'en retourne chez lui vaincu par Grimberge et que tout continue, comme si le combat n'avait eu aucune importance. Le seul ordre qui

\footnotetext{
24 «Vilain and courtois functions as a moral polarization (that of high and low, or good and not good) that is projected in terms of a class opposition » («Le couple vilain et courtois fonctionne selon une polarité morale (opposant le haut et le bas, ou le positif et le négatif) conçue comme une opposition entre classes sociales »), Kathryn GRAVDAL, "Vilain et courtois». Transgressive Parody in French Literature of the Twelfth and Thirteenth Centuries, Lincoln et London, University of Nebraska Press, 1989, p. 64.

${ }^{25}$ Cf. Le Vilain asnier, Le Pez au vilain, La Crote...
} 
motive la narration du poème semble ainsi être un ordre cyclique, qui va d'une naissance à une autre et d'un mariage à un autre.

Cette dynamique cyclique, associée à la thématique carnavalesque, peut éclairer sous un autre angle la fonction de l'excrémentiel dans ce texte. Pour Bakhtine en effet, le rabaissement carnavalesque, qui consiste à dégrader ce qui est élevé en l'abaissant vers la terre, a pour fin la régénération : il est à la fois une mort et une renaissance. Or l'excrément, auquel l'anthropologie prête l'ambivalence qui caractérise la sphère du «pourri ${ }^{26}$, semble particulièrement adapté à cette problématique : s'il renvoie symboliquement au cadavre en raison de sa puanteur et permet ainsi d'interpréter les affronts de Grimberge comme les formes atténuées d'une mise à mort, il est aussi le fumier qui fertilise la terre, comme le dit galamment Audigier à sa belle :

«[...] ice m'est bel

que nos fumerons bien nostre prael.

La fumee m'en monte jusqu'au cervel

et la fairor m'en vient jusqu' au musel. » ${ }^{27}$

Les défécations incessantes des personnages féminins renverraient à leur fertilité symbolique et le couple Raimberge/Grimberge pourrait, dans cette perspective, incarner les deux faces du pouvoir menaçant de la fécondité féminine.

On peut aussi aller plus loin et voir derrière la thématique carnavalesque un motif folklorique, voire un substrat païen. Pour Lucia Lazzerini $^{28}$, Audigier représenterait en fait la parodie carnavalesque du motif folklorique de l'initiation, dont on retrouve le thème traditionnel de l'avalement. Le poème accorde en effet une large place à l'adoubement du

\footnotetext{
${ }^{26}$ Cf. Louis-Vincent ThOMAs, Le Cadavre. De la biologie à l'anthropologie, Bruxelles, Éditions Complexe, 1980, p. 98 sqq.

${ }^{27}$ Audigier, éd. cit., v. 492-495.

${ }^{28}$ Cf. Lucia LAZZERINI, «Audigier». Poema eroicomico antico-francese, Florence, Sansoni, 1985.
} 
héros, cérémonie officielle qui prépare le véritable rite de passage tel qu'il se présentera dans le dernier combat avec Grimberge :

La vielle s'en torna qui fu enflee, enprés Audigier cort geule baee. [...] aussi le tranglouti com une oublee. Et quant ele senti qu'el fu enflee, a terre s'acroupi, li cus li bee. Audigier s'en oissi, criant : «Outree ! » A tent ez vo Bougise toute aïriee et vint a Audigier sanz demoree.

$$
[\ldots]
$$

et, quant il fu cheüz geule baee, si li pisse el visaige de randonnee :

le cors en ot moillié et l'eschinee. ${ }^{29}$

Littéralement englouti et déféqué par Grimberge, Audigier retournerait dans une mort symbolique au ventre maternel tandis que Bougise, en l'arrosant d'urine, le ramènerait tout aussi symboliquement à la vie $\mathrm{ie}^{30}$. Luciana Borghi Cedrini étaye cette hypothèse folkloriste en montrant que, comme dans Le Pez au vilain de Rutebeuf, le pet est dans Audigier le représentant de l'âme ${ }^{31}$; en effet, il faut attendre la mort de Turgibus sur un tas de fumier pour que naisse Audigier, conçu parmi les flatulences et mis au monde dans les excréments, comme si l'âme de son père s'incarnait en lui. Cette hypothèse semble d'autant plus intéressante que parmi les assaillants de Turgibus se trouvent des bousiers ${ }^{32}$ qui, dans certaines

\footnotetext{
${ }^{29}$ Audigier, éd. cit., v. 338-354.

${ }^{30}$ Le baptême avait déjà eu lieu dans l'urine.

${ }^{31}$ Cf. Luciana Borghi CEDRINI, La Cosmologia del villano. Secondo testi extravaganti del Duecento francese, Alessandria, Edizioni dell'Orso, 1989. Cf. aussi Jean-Claude AubAILly, «Le fabliau et les sources inconscientes du rire médiéval », Cahiers de Civilisation Médiévale, XXX (1987), p. 105-117. Le motif du pet trouverait son origine dans le thème de la circulation des souffles assimilés aux âmes, que l'on retrouve dans le mythe de l'ours, être psychopompe qui libèrerait les âmes au moyen d'un pet lors de sa déshibernation. Ce motif serait ainsi une résurgence et une désacralisation du culte primitif de l'ours, à l'origine aussi du vent de la fête des fous.

32 « escharboz l'assaillirent en un fumier », Audigier, éd. cit., v. 110.
} 
sociétés, sont directement liés à la mort et à la régénération ${ }^{33}:$ il y aurait ainsi dans Audigier des résurgences mythiques d'un substrat païen. Représentative de la filiation qui existe entre paganisme et carnaval, la scatologie aurait donc pour fonction de représenter le cycle sans fin de la vie et de la mort. Comme le dit Lucia Lazzerini, «l'excrément, métaphore unificatrice de l'initium (le inter faeces et urinam nascimur augustinien) et de l'exitus (comme destination inéluctable de la putréfaction), devient le symbole de la vitalité humaine: le fumier est le centre de l'univers d'Audigier, le théâtre des principaux évènements qui s'y jouent ${ }^{34}$. Mais pourquoi avoir gardé la forme aussi manifeste d'une chanson de geste pour une vision du monde qui ne correspond pas à ce genre alors que le fabliau, avec son jeu sur la dialectique entre ordre et désordre, parait plus adapté à l'expression d'un mundus inversus aux résonances métaphysiques?

\section{Une poétique inédite}

Une première hypothèse consisterait à voir dans la conservation d'une forme épique, qui fait ressortir la discordance d'un contenu moins noble, un moyen facile de provoquer un rire libérateur né d'un sentiment de décalage. Dans la mesure où Audigier semble aborder des problématiques plus graves qu'il n'y paraît, le rire permet en effet de dédramatiser des

\footnotetext{
${ }^{33}$ Dominique G. Laporte rappelle que le bousier apparaît dans de nombreux mythes de la Création, par exemple en Égypte où « le scarabée qui semble surgir vivant de la fiente ou de la boue était crédité du pouvoir de renaître de lui-même après sa mort ». Cette conception aurait même pu pénétrer la pensée chrétienne puisque saint Ambroise aurait appelé le Christ «bonus scarabeus» ou «scarabeus in cruce». Cf. John Gregory BOURKe, Les Rites scatologiques, Dominique G. LAPORTE (éd.), Paris, PUF, 1981 (1 $1^{\text {ère }}$ éd. américaine 1891), p. 24.

34 «L sterco, metafora unificante dell'initium (l'agostino «inter faeces et urinam nascimur ») e dell'exitus (come ineluttabile destino di putrefazione) diventa il simbolo della vicenda umana : il letamaio è il centro dell'universo di Audigier, il teatro degli eventi cruciali della storia », Lucia LAZZERINI, op. cit., p. 73-74.
} 
enjeux trop lourds. Herman Braet $^{35}$ a montré comment cette rhétorique du décalage repose sur l'utilisation à contre-emploi de formules épiques traditionnelles, tout aussi identifiables qu'incongrues. Ainsi, lorsque Audigier revient barbouillé d'excréments, Raimberge rayonnante lui fait remarquer : «Du vis et de la chiere bien resanblez / Voz peres Turgibus qui tant fu ber ${ }^{36}$. Cet attachement à dissocier fond et forme conduit légitimement à se demander si, en plus d'être un moyen commode pour provoquer le comique nécessaire à la réception du texte, le jeu de décalage n'interrogerait pas aussi les rapports entre la littérature et le monde, entre le mot et la chose, comme c'est si souvent le cas dans les fabliaux. Instaurer un décalage constant entre des formules et ce qu'elles sont censées exprimer ressemble fort en effet à une manière de les dénoncer comme creuses, vidées de leur sens. On peut, avec Herman Braet, se demander « si à force de nous désarçonner de la sorte, cet écrivain ne vise pas autant le cliché que le sujet [...]. Les formules ne sont plus ce qu'elles étaient, les vocables ne signifient plus ce qu'ils signifiaient. En jouant sur l'écart entre les mots et l'idée, l'auteur d'Audigier dénonce un contrat ${ }^{37}$. Le décalage renvoie la formule littéraire à son artificialité, ce qui revient aussi à renvoyer les valeurs qu'elle reflète à un monde d'illusion : en confrontant son lecteur à l'artificialité du langage, l'auteur détruit le pacte narratif.

Cependant, le jeu sur le langage ne semble pas du tout atteindre la profondeur et la complexité qu'il acquiert dans les fabliaux. En effet, si l'on retrouve bien dans la description redondante et complaisante des bourbiers de Cocuce ce qui ressemble à l'emballement verbal des fabliaux,

\footnotetext{
${ }^{35}$ Herman BRAET, «Audigier, de la dissonance comme moyen de dérision », in Jean DUFOURNET (dir.) «Si a parlé par moult ruiste vertu». Mélanges de littérature médiévale offerts à Jean Subrenat, Paris, Champion, "Colloques, congrès et conférences sur le Moyen Âge », 1, 2000, p. 83-90.

${ }^{36}$ Audigier, éd. cit., v. 437-438. Comme le dit Hermann Braet «le lecteur aura reconnu l'épithète, usée jusqu'à la corde » (Herman BRAET, «Audigier, de la dissonance... », art. cit., p. 85).

${ }^{37}$ Ibid., p. 90.
} 
les composantes obscènes du texte ne donnent pas lieu à un jeu en tant que tel : seul le décalage est amusant. Pour Eric Hicks « il s'agit moins d'une écriture proprement scandaleuse que d'un texte parfaitement débile [...] au niveau de l'histoire de Toto », « qui choque davantage l'intelligence que la morale, ou même la décence ${ }^{38}$. En effet, à l'inverse de ce qui se passe dans les fabliaux, les mots scatologiques et les scènes ordurières ne sont pas tant les objets d'une manipulation jouissive que des éléments de décor, presque épuisés par leur caractère répétitif et monotone. Mais si les thèmes scatologiques n'ont pas de valeur littéraire, pourquoi apparaissent-ils dans un texte qui se moque si nettement des pratiques de la chanson de geste ? On pourrait penser que la forme épique serait moins mise en cause qu'elle ne se révèlerait un moule pratique pour des motifs populaires. Selon Éric Hicks en effet « la réponse qui consiste à trouver une sortie du côté de la parodie ne résiste pas à l'expérience du texte » car «s'il y a rencontre du style élevé et du bas-étage, celui-là n'est pas subverti par celui-ci. Au contraire, la diction épique sert à rendre soutenable un discours perçu comme immonde $»^{39}$. On peut effectivement se demander si l'exhibition du corps dans ses fonctions les plus basses à l'intérieur d'un monde dont toutes les composantes sont véritablement grotesques ne provoque pas plus sur l'auditoire un effet de dégoût que l'exaltation que voudrait y voir Bakhtine. La forme épique serait-elle une manière de tempérer un discours extrêmement pessimiste et insoutenable, tel qu'il se présente dans le contemptus mundi? Il faut bien avouer qu'Audigier ressemble fort peu à un manifeste ascétique. S'il est indéniable qu'il provoque sûrement du dégoût, celui-ci est désamorcé par le rire. La poétique du texte, fondée sur l'ambivalence entre le rire et l'angoisse que permet le décalage entre forme

\footnotetext{
${ }^{38}$ Éric HICKS, «Pourquoi Audigier? », in Danielle BUSCHINGER et André CREPIN (dir.) Amour, mariage et transgressions au Moyen Âge. Actes du colloque des 24-25-26 et 27 mars 1983, Amiens, Göppingen, A. Kümmerle, 1984, p. 284-285.

${ }^{39}$ Ibid., p. 286-287.
} 
et matière, reflète donc l'ambivalence de ce qu'il met en scène : le passage incessant entre les deux réalités incompatibles mais néanmoins complémentaires que sont la vie et la mort.

Toutefois, quelle que soit l'interprétation que l'on fasse de la réduction manifeste du vivant au bas corporel que met en œuvre Audigier, la question qui se pose, comme le rappelle Éric Hicks, «n'est pas celle de l'existence de ces fantasmes, mais celle de leur surgissement sous forme littéraire. Pourquoi ce parchemin gâté ? ${ }^{40}$. S'il nie à Audigier toute valeur littéraire, Éric Hicks parvient pourtant lui aussi au constat d'une rupture, qu'à la différence d'Herman Braet il ne place pas seulement au niveau littéraire : ce ne serait pas tant la littérature que l'institution du langage ellemême que mettrait en cause Audigier. En effet, tandis que la diction épique maintiendrait «comme en bruit de fond un minimum de communication discursive », Audigier constituerait, moins par son caractère ordurier que par son absurdité, un « refus de communication », une « injure du poète à son public $»^{41}$. En dessinant à l'orée de son texte l'image d'un auditeur destiné à demander grâce comme s'il ployait sous les défécations («Tel conte d'Audigier qui en set pou, / mais ge vos en dirai trusqu'a harou $\gg^{42}$ ), l'auteur met en exergue le pouvoir évocateur du langage qui aurait moins alors une fonction de communication qu'une fonction de création. On peut d'ailleurs noter que le texte met lui-même en scène ce pouvoir créateur du langage à travers le passage du mot à la chose :

« Audigier, dit Grinberge, bouse vos di.

De trois de mes estrons et un demi

Vos desgeüneroiz demain matin

Si baiseroiz mon cul et l'aubatri. » ${ }^{43}$

\footnotetext{
${ }^{40}$ Ibid., p. 286.

${ }^{41}$ Ibid., p. 288.

${ }^{42}$ Audigier, éd. cit., v. 1-2.

${ }^{43}$ Audigier, éd. cit., v. 321-324.
} 
D'un vers à l'autre, l'injure est devenue réalité tangible. Ainsi, dans le temps même où le détournement des formules épiques le représente comme artificiel, le langage est envisagé dans ses virtualités créatrices.

Ce constat inciterait à penser qu'Audigier pose les mêmes questions que les fabliaux en interrogeant véritablement le langage comme représentation. Cependant, contrairement aux fabliaux qui se construisent sur cette problématique et l'exploitent jusqu' au vertige, Audigier ne semble que l'esquisser dans une forme littéraire moins élaborée. Or, si l'on reprend les estimations de Jodogne ${ }^{44}$, la version d'Audigier qui nous est parvenue daterait de la fin du XII ${ }^{\mathrm{e}}$ siècle ou du tout début du XIII ${ }^{\mathrm{e}}$, mais les allusions d'Aiol ${ }^{45}$ portent à croire qu'une version antérieure à 1160 a dû exister. Peut-être alors ne serait-il pas téméraire de supposer qu'Audigier fait état de l'affranchissement progressif d'une tradition déjà présente dans les chansons de geste ${ }^{46}$ et qui aurait pris assez d'importance pour s'en détacher dans la seconde moitié du XII ${ }^{\mathrm{e}}$ siècle. Soit qu'elle corresponde déjà dans la chanson de geste à une conception de l'ordre du monde qui aurait eu besoin d'être remotivée, soit qu'elle se trouve seulement ensuite investie de cette problématique, elle aurait pu progressivement devenir matière à littérature et finalement trouver dans le genre du fabliau, qui s'épanouit justement à partir de la fin du $\mathrm{XII}^{\mathrm{e}}$ siècle, la forme littéraire la plus adaptée à ses enjeux.

\footnotetext{
${ }^{44}$ Omer JodoGNE, « Audigier et la chanson de geste... », art. cit., p. 500-502.

${ }^{45}$ Aiol fait en effet plusieurs références explicites à Audigier. La bouchère Hersent, comme nous l'avons vu, ressemble fortement à Grimberge, une bourgeoise porte le nom de «Raiborc» ou «Raiborghe» (Aiol, éd. cit., v. 2740 et 2744) et, surtout, c'est directement à la famille d'Audigier que les badauds comparent Aiol revêtu de son piètre équipement : "Furent ces arme[s] faite[s] en vos resné ? / Fu Audengier[s] vos peres qui tant fu ber / Et Rai[m]bergue vo mere o le vis cler?» (v. 991-993). Ces allusions ont été remarquées par Omer Jodogne, qui en conclut qu'une première version perdue d'Audigier a dû influencer Aiol (Omer JODOGNE, «Audigier et la chanson de geste... », art. cit., p. 507).

${ }^{46}$ Voir, par exemple, la Chanson de Guillaume, datée des environs de 1140.
} 
Le mélange incongru de chanson de geste et de fabliau que constitue Audigier conduit donc à regarder d'un peu plus près les différents genres dont il semble procéder. D'une part, si le thème scatologique entraîne le rapprochement avec le fabliau, le fait qu'il prenne une forme épique rappelle qu'il est déjà présent dans la chanson de geste. Or, puisque le rapprochement avec le fabliau révèle sa composante carnavalesque et ses liens avec un substrat païen, on peut à juste titre se demander si c'est ce même substrat que l'on trouve à l'œuvre dans certains poèmes épiques, ou s'il s'est greffé sur des motifs qui lui sont d'abord étrangers. Un personnage comme Rainouart par exemple, qui prend à rebours tous les codes chevaleresques mais qui confirme les valeurs épiques plus qu'il ne les transgresse, invite à faire le lien entre carnaval et structure trifonctionnelle et donc à observer la superposition et l'évolution de différents systèmes idéologiques au sein des œuvres médiévales ${ }^{47}$.

D'autre part, la relative simplicité d'Audigier met en valeur l'art littéraire de nombreux fabliaux qui partagent avec le poème leur thématique : par comparaison, notre texte révèle à quel point le comique scatologique des fabliaux, parfaitement intégré à un schéma narratif fondé sur la ruse et le rebondissement, participe d'un jeu sur l'illusion et la représentation. L'interrogation réflexive sur la littérature n'est qu'une ébauche dans Audigier. Cependant, «mais ge vos en dirai trusqu'a

${ }^{47}$ Comme l'a montré Joël Henri Grisward (cf. Archéologie de l'épopée médiévale, Paris, Payot, 1981 et «La naissance du couple littéraire Vivien et Rainouart», Medioevo Romanzo, XXI (1997), p. 441-456), la deuxième fonction indo-européenne, celle des guerriers, oppose les héros d'Odhin, qui présentent des affinités avec la première fonction magico-religieuse, et les héros de Thôrr qui s'orientent vers la troisième fonction nourricière. De toute évidence, Rainouart appartient à la deuxième catégorie, ce qui fait de son goût pour la nourriture, le sommeil et la boisson des caractéristiques structurelles du personnage. Cependant, la structure trifonctionnelle semble chez Rainouart être recouverte par l'idéologie carnavalesque, le personnage mettant lui-même en œuvre au cours de sa carrière une dynamique cyclique de dégradation et de résurrection. Ce constat invite donc d'une part à remarquer que les œuvres médiévales combinent plusieurs héritages idéologiques et d'autre part à s'interroger sur les conditions et les possibilités de tels recouvrements. 
harou ${ }^{48}$ établit, comme dans Jouglet, un lien entre matière fécale et parole : à la diarrhée constante du personnage répond la logorrhée du narrateur, ce qui tournerait la parole du côté des déjections corporelles et constituerait un jugement de valeur réflexif sur une littérature qui fait du rire et du plaisir ses enjeux, dans laquelle les jongleurs sont, significativement, récompensés de crottes de chien.

${ }^{48}$ Audigier, éd. cit., v. 2. 